\title{
Amino Acids in Metabolomics: Perspective for the Use of Regulatory Effects of Free Amino Acids in the Creation on their Basis of Infusion Solutions
}

\author{
Karavay P.A ${ }^{1 *}$, Leonid I. Nefyodov ${ }^{2}$, Karavay N.L ${ }^{3}$ \\ ${ }^{1}$ Department of Oncology, Grodno State Medical University, Republic of Belarus, Belarus \\ ${ }^{2}$ Department of Biochemistry, Yanka Kupala Grodno State University, Republic of Belarus, Belarus \\ ${ }^{3}$ Department of Hyperbaric Oxygenation, Grodno State Medical University, Republic of Belarus, Belarus
}

*Corresponding author: Karavay P.A, Department of Oncology, Grodno State Medical University, Belarus, E-mail:

1.nefyodov@mail.ru

Citation: Karavay, P.A., et al. Amino Acids in Metabolomics: Perspective for the Use of Regulatory Effects of Free Amino Acids in the Creation on their Basis of Infusion Solutions (2016) Int J Hematol Ther 2(2): 1- 2.

\begin{abstract}
The creation methodology of pathogenetic compositions of amino acids and their derivatives on the basis of their physiological concentration for practical application of regulatory effects of these substances was determined in this study.
\end{abstract}

Keywords: Free amino acids; Regulatory effects; Infusion solutions
Received Date: April 3, 2016

Accepted Date: May 19, 2016

Published Date: May 26, 2016

DOI: $10.15436 / 2381-1404.16 .10$

\section{Introduction}

Amino acid profile indexes are being created that allow early detection of diseases that would provide time for intervention before irreversible damage has occurred. Thus, amino acid profiles represent biomarkers for diseases or from deviations from a normal state of health. Our array technology will play an important role in metabolomics in biomarker discovery, clinical medicine, as well as in other stages of drug discovery and development (e.g. target discovery, mechanism of action or predicting toxicity ${ }^{[1-6]}$.

To date, there are three main levels of exploitation of bio- chemical (metabolic) properties of amino acids and their derivatives in clinical practice:

The use of amino acids or multicomponent mixtures of amino acids (mainly, essential, combined with vitamins and trace elements) for replacement therapy or shortfall of essential nutrients and protein ${ }^{[1]}$.

1. The use of drugs on the basis of individual amino acids or their compositions, developed based on the additive functional and metabolic action, in which the "exploited" pharmacological activity (effects of activation of redox processes, reactions of energy metabolism and neutralization of xenobiotics compounds) of this class ${ }^{[2]}$.

2. However, the use of certain levels of L-amino acids or their compositions or shortfall implement direct pharmacological effects practically ignored their regulatory effects on metabolic processes and key metabolic reactions.

3. To understand the metabolic processes and vital functions of the regulatory effect of amino acids, which mani-fests itself under natural or near concentrations of these compounds in body fluids and tissues ${ }^{[3-5]}$. it is obvious that the effective use of L-amino acids or their derivatives for metabolic correction and directional changes in me-tabolism in pathological or extreme conditions limited accumulation of information about theme chanisms of regulatory effects of the compounds tested at concentrations comparable to their physiological (endogenous) level ${ }^{[6]}$.

\section{Materials and Methods}

Our development methodology is based on:

1. Study of physiological concentrations of free amino acids, their derivatives, precursors and metabolites, as well as, biochemical marker parameters in healthy donors and patients with various pathologies ${ }^{[1,6]}$.

2. Creating a unified database for the studied parameters, construction of empirical mathematical model consisting of patho-

Copyrights: (C) 2016 Karavay P.A. This is an Open access article distributed under the terms of Creative Commons Attribution 4.0 International License. 
genic markers specific pathology and amino acid profiles ${ }^{[2,6]}$.

3. Specialized development of new formulations of the compositions of infusion solutions of amino acids and their derivatives $^{[1,3,6]}$.

\section{Results and Discussion}

Numerous results of determination of amino acids and their derivatives in human body fluids and tissues ${ }^{[4]}$ allowed systematization of the accumulated data and identified areas for exploitation of their metabolic effects, primarily, in laboratory diagnostics and application in clinical practice as drugs ${ }^{[5]}$.

In view of the fact that the free amino acids are represented by a wide range of related chemical structure and metabolic transformations of compounds that forms in the body fluids and tissues, amino acid found proved that quantification of their pool contributes to the diagnosis of various diseases, including hepatobiliary pathology, cardiovascular and immune systems, oncological causes, cerebrovascular pathology, alcoholism and diabetes $^{[6]}$. It turned out that the vast majority of the diagnostic values of the group have shifts in the level of functional and metabolic related amino acids and their derivatives, and as such no specific changes in the concentrations of individual compounds of this class is observed.

Metabolic related amino acids and their derivatives had the nature of the amino acid profiles of the body fluids and tissues of animals and humans when compared with the use of multivariate analysis and mathematical modeling. At the same time, it was convincingly demonstrated that the removal or correction of the intermediate metabolic changes can be achieved using individual amino acids and their derivatives, or a combination thereof as universal natural bioregulators - compounds that has direct effect on the mechanisms of cellular metabolism in physiological concentrations ${ }^{[2,7,9]}$.

To date, there are evidences of the importance of not only amino acids as building blocks for protein synthesis, but regulators of gene expression at the level of mRNA translation by mTOR-dependent mechanism, signaling molecules and biological response modifiers, as well as, precursors of a wide range of bioregulators, which play a key role in the integration of major metabolic fluxes ${ }^{[8,10,11]}$.

Based on the positions of metabolomics, the amino acid pool of biological fluids and tissues found free amino acids evaluated as a single information unit, which is a kind of "chemical projection" of the genome, proteome realized through this approach not only develops ideas about the pool of amino acids as a dynamical system generated receipt of them from outside, but also due to endogenous synthesis, transport, degradation and excretion, and allows the identification of "key points" intermediate metabolic equilibrium shift that may reflect ratios at the individual levels of endogenous amino acids and related species (metabolic related) compounds.
Our proposed methodology for developing new formulations multicomponent infusion solutions based on amino acids and related compounds for the correction occurring in various diseases of the metabolic imbalance is based on the application of research results regularities of formation of amino acid found in biological fluids and tissues in various pathological conditions. The composition and quantity of highly purified amino acids in these infusion solutions should be determined primarily by their physiological (regulatory) concentrations, which distinguishes them from the traditionally used amino acid solutions for parenteral nutrition, where the content of their components is calculated based on the daily needs of the human body in them without due consideration of regulatory actions administered to compounds ${ }^{[2,6]}$.

\section{Conclusion}

The methodology of development of new compoundings of multicomponent infusion solutions offered by us on the basis of amino acids and the related connections is intended for correction of the metabolic imbalance arising at various diseases based on application of results of research of regularities of formation of amino acid found in biological liquids and tissues $o$ individuals at most various pathological states (more than 9000 surveyed ${ }^{[1-3,6]}$.

\section{References}

1. Nefyodov, L.I. Taurine (chemistry, biochemistry, medical use) (1999) Grodno 140

2. Nefyodov, L. Amino Acids and their derivatives (chemistry, biochemistry, medical use) (2001) Proc of Internat Symp Grodno 124.

3. Nefyodov, L.I., Karavay, P.A., Karavay, N.L. Regulatory action of free amino acids and development on the basis of highly of substances infusion solutions with pathogenetic deterministic composition. (2014) Laboratory diagnosis Eastern Europe 3: 111-115.

4. Holden, J.T. Amino Acid Pools. (1962) Elsevier, Amsterdam 815.

5. Lubec, C. Amino Acids (Chemistry, Biology, Medicine) (1990) Escom New York 1196.

6. Nefyodov, L.I. The results of biochemical research and development of nitrogen-containing compounds of natural origin: methodology of exploitation of biological properties as universal natural regulators of metabolism and drugs. (2010).

7. Ihata, Y., Miyagi, E., Numazaki, R. Amino acid profile index for early detection of endometrial cancer: verification as a novel diagnostic marker. (2013) Int J Clin Oncol 19(2): 364-372.

8. Fafournoux, P., Bruhat, A., Jousse, C. Amino acid regulation of gene expression. (2000) BioChem J 351(1): 1-12.

9. Nefyodov, L.I. Target-oriented regulation of metabolic equilibrium by amino acids and strategy of their application as drugs with directional effects 37 Polish Biochemical Society Congress, Torun (2001).

10. Meijer, A. Amino acids as regulators and components of nonproteinogenic pathways. (2003) J Nutr 6(1): 2057S-2062S.

11. Bruhat, A., Cherasse, Y., Chaveroux, C., et al. Amino acids as regulators of gene expression in mammals: molecular mechanisms. (2009) Biofactors 35(3): 249-257.
Ommega Online Publisher

International Journal of Hematology \& Therapy

Short Title : Int J Hematol Ther
ISSN: 2381-1404

E-mail : hematology.therapy@ommegaonline.org website: www.ommegaonline.org 